\title{
BANACH SPACES OF LIPSCHITZ FUNCTIONS AND VECTOR-VALUED LIPSCHITZ FUNCTIONS ${ }^{1}$
}

\author{
BY J. A. JOHNSON ${ }^{2}$
}

\author{
Communicated by Bertram Yood, May 16, 1969
}

Introduction. Given a metric space $(S, d)$, and a Banach space $E$, let $\operatorname{Lip}_{E}(S, d)$ denote the vector space of bounded functions $f: S \rightarrow E$ such that

$$
\|f\|_{d}=\sup \left\{\|f(s)-f(t)\| d^{-1}(s, t) \mid s \neq t\right\}
$$

is finite. Let $\|\cdot\|_{\infty}$ denote the sup-norm. Then $\operatorname{Lip}_{E}(S, d)$ endowed with $\|\cdot\|=\max \left(\|\cdot\|_{\infty},\|\cdot\|_{d}\right)$ is a Banach space. $\operatorname{lip}_{E}(S, d)$ denotes the closed subspace of functions $f$ such that

$$
\lim _{a(s, t) \rightarrow 0}\|f(s)-f(t)\| d^{-1}(s, t)=0 .
$$

When $E$ is the set of real or complex numbers, we drop the subscript and write $\operatorname{Lip}(S, d)$ and $\operatorname{lip}(S, d)$.

In this paper we examine the Banach space properties of $\operatorname{lip}(S, d)$ and $\operatorname{Lip}(S, d)$ and extend some known results. Details and proofs of results presented here will appear elsewhere.

The author wishes to express his thanks to Professor D. R. Sherbert for his advice in preparing this paper.

1. Weak completeness and extreme points.

Theorem 1.1. Let $(S, d)$ be any metric space. A sequence $\left\{f_{n}\right\}$ in $\operatorname{lip}(S, d)$ is weakly Cauchy if and only if it is bounded and every sequence $\left\{s_{m}\right\}$ in $S$ has a subsequence $\left\{s_{m_{i}}\right\}$ such that $\lim _{n \rightarrow \infty} \lim _{i \rightarrow \infty} f_{n}\left(s_{m_{i}}\right)$ exists.

Corollary 1.2. If $(S, d)$ is compact, $\left\{f_{n}\right\}$ is weakly Cauchy if and only if it is bounded and $\lim _{n \rightarrow \infty} f_{n}(s)$ exists for each $s \in S$.

If $0<\alpha \leqq 1$ and $d$ is a metric, so is $d^{\alpha}$. We frequently consider $\operatorname{lip}\left(S, d^{\alpha}\right)$ for $0<\alpha<1$, since this space separates points.

Theorem 1.3. Let $(S, d)$ be any metric space and $0<\alpha<1$. Then neither $\operatorname{Lip}\left(S, d^{\alpha}\right)$ nor $\operatorname{lip}\left(S, d^{\alpha}\right)$ is weakly sequentially complete unless $S$ is a finite set.

1 This paper is a portion of the author's doctoral dissertation submitted to the University of Illinois.

2 This research was supported in part by the National Science Foundation. 
The proof of 1.3 consists in using 1.1 to show that if $\operatorname{lip}\left(S, d^{\alpha}\right)$ is weakly sequentially complete, it is isomorphic with the space of bounded functions on $S$.

In [6] Jenkins characterized the extreme points of the dual ball of $\operatorname{lip}\left(S, d^{\alpha}\right)$ when $0<\alpha<1$ and $(S, d)$ is compact. This generalized a result of deLeeuw [3]. He also gave a partial description of the extreme points of the dual ball of $\operatorname{Lip}\left(S, d^{\alpha}\right)$. We state this latter result below for reference.

If $f \in \operatorname{Lip}\left(S, d^{\alpha}\right)$ and $W=\{(s, t) \in S \times S \mid s \neq t\}$, define $\tilde{f}(s, t)=$ $(f(s)-f(t)) / d^{\alpha}(s, t)$ if $(s, t) \in W$ and $\tilde{f}(s)=f(s)$ if $s \in S$. Then $\tilde{f} \in C$ $(S \cup W)$, the bounded continuous functions on $S \cup W$. Let $S$ be compact. We can extend each $\tilde{f}$ to an element $\hat{f}$ in $C(S \cup \beta W)$, where $\beta W$ is the Stone-Cech compactification of $W . f \rightarrow \hat{f}$ is a linear isometry. The result of Jenkins states that the extreme points of the dual ball are precisely the functionals of the form $f \rightarrow \lambda f(s)$ and $f \rightarrow \lambda \tilde{f}(s, t)$, for $s \in S, 0<d(s, t)<2$ and $|\lambda|=1$, plus some subset $Q$ of functionals of the form $f \rightarrow \lambda \hat{f}(\omega)$ where $\omega \in \beta W \sim W,|\lambda|=1$. The nature of $Q$ was left an open problem in [6]. Using 1.3 and Rainwater's theorem (see [7]), we get

THEOREM 1.4. $Q \neq \varnothing$ unless $S$ is a finite set.

\section{Compactness.}

Lemma 2.1. Let $(S, d)$ be any metric space and $F$ a bounded subset of $\operatorname{Lip}(S, d)$. Then $\tilde{F}=\{\tilde{f} \mid f \in F\}$ is equicontinuous at each point of $S \cup W$.

TheOREM 2.2. Let $(S, d)$ be compact. A subset $F$ of $\operatorname{lip}(S, d)$ is relatively compact if and only if

$$
\lim _{\Omega(s, t) \rightarrow 0} d^{-1}(s, t)|f(s)-f(t)|=0 \quad \text { uniformly for } f \in F .
$$

COROLlaRy 2.3. The unit ball of $\operatorname{Lip}\left(S, d^{\beta}\right)$ is compact in $\operatorname{lip}\left(S, d^{\alpha}\right)$ for $0<\alpha<\beta \leqq 1$.

THEOREM 2.4. The following are equivalent:

(a) $(S, d)$ is precompact.

(b) The unit ball of $\operatorname{Lip}\left(S, d^{\alpha}\right), 0<\alpha \leqq 1$, is compact for the sup-norm topology.

(c) The unit ball of $\operatorname{Lip}\left(S, d^{\beta}\right)$ is compact in $\operatorname{lip}\left(S, d^{\alpha}\right)$ for $0<\alpha<\beta \leqq 1$.

REMARK. A characterization of the weakly relatively compact subsets of $\operatorname{lip}(S, d)$ similar to 2.2 but involving quasi-equicontinuity can be given. 
3. $\operatorname{Lip}_{E^{\prime}}(S, d)$ as a dual space and $\operatorname{Lip}\left(S, d^{\alpha}\right)$ as a second dual. Several results have appeared which recognize various spaces of Lipschitz functions as dual spaces (see [1] and [8]). By using a theorem due to Dixmier [4], we obtain the following result.

THEOREM 3.1. For any metric space $(S, d)$ and any Banach space $E$, $\operatorname{Lip}_{E}(S, d)$ is a dual space whenever $E$ is.

Considering $\operatorname{Lip}(S, d)$ and $\operatorname{Lip}_{E}(S, d)$ as dual spaces, we can characterize weak* sequential convergence.

Theorem 3.2. A sequence $\left\{f_{n}\right\}$ in $\operatorname{Lip}_{E}(S, d)$ converges weak $k^{*}$ to zero if and only if it is bounded and $\left\{f_{n}(s)\right\}$ converges weak* to zero in $E$ for each $s \in S$. If $(S, d)$ is compact, a sequence $\left\{g_{n}\right\}$ converges weak $k^{*}$ to zero in $\operatorname{Lip}(S, d)$ if and only if it is bounded and converges uniformly to zero.

For the rest of this paper, unless otherwise stated, assume that every closed bounded subset of $(S, d)$ is compact. Let $\operatorname{lip}^{0}\left(S, d^{\alpha}\right)$ denote the space of functions in $\operatorname{lip}\left(S, d^{\alpha}\right)$ that vanish at infinity. The main result of [6] is that for real-valued functions, the bidual of $\operatorname{lip}^{0}\left(S, d^{\alpha}\right)(0<\alpha<1)$ is $\operatorname{Lip}\left(S, d^{\alpha}\right)$. For complex-valued functions, an extra hypothesis on $(S, d)$ was required. (This generalized the original work of deLeeuw [3] along these lines.) By using 3.1, we are able to give a different proof of this result which shows the extra hypothesis in the complex case to be unnecessary.

4. $\operatorname{Lip}_{E^{\prime \prime}}(S, d)$ as a second dual. Given a Banach space $E$, let $E^{\prime}$ and $E^{\prime \prime}$ denote its dual and bidual respectively. Since $\operatorname{Lip}_{E^{\prime \prime}}\left(S, d^{\alpha}\right)$ is a dual space, it is natural to ask if it is the bidual of $\operatorname{lip}_{E}^{0}\left(S, d^{\alpha}\right)$, $0<\alpha<1$. ( $f: S \rightarrow E$ is said to vanish at infinity if $|f|$ does, where $|f|(s)=\|f(s)\|)$.

In the scalar-valued case the canonical mapping of $\operatorname{lip}^{0}\left(S, d^{\alpha}\right)^{\prime \prime}$ into $\operatorname{Lip}\left(S, d^{\alpha}\right)$ is shown to be one-to-one by showing that the point evaluations span a dense subspace of $\operatorname{lip}^{0}\left(S, d^{\alpha}\right)^{\prime}$. We are able to extend this to the vector-valued case by defining a canonical map $\Lambda: \operatorname{lip}_{E}^{0}\left(S, d^{\alpha}\right)^{\prime \prime} \rightarrow \operatorname{Lip}_{E^{\prime \prime}}\left(S, d^{\alpha}\right)$ in an analagous way and showing that functionals of the form $f \rightarrow\left\langle f(s), x^{\prime}\right\rangle, s \in S, x^{\prime} \in E^{\prime}, f \in \operatorname{lip}_{E}^{0}\left(S, d^{\alpha}\right)$, span a dense subspace of $\operatorname{lip}_{E}^{0}\left(S, d^{\alpha}\right)^{\prime}$. This is done by use of vectorvalued measures.

At this point, the methods used in the scalar-valued case fail because of an inability to extend vector-valued Lipschitz functions in a norm-preserving way. To bypass this, the theory of tensor products of Banach spaces is employed. The following result is vital for this purpose. 
THEOREM 4.1. For any metric space $(S, d)$ and Banach space $E$, $\operatorname{Lip}_{E^{\prime}}(S, d)$ is isometrically isomorphic with the space $\mathfrak{L}(E, \operatorname{Lip}(S, d))$ of bounded linear maps from $E$ into $\operatorname{Lip}(S, d)$.

REMARK. When $(S, d)$ is compact, the isometry in 4.1 identifies $\operatorname{lip}_{E^{\prime}}(S, d)$ with the compact operators having range in $\operatorname{lip}(S, d)$.

Now, if $V$ denotes the closed linear span in $\operatorname{Lip}(S, d)^{\prime}$ of the point evaluations, then the tensor product $E \otimes V$ of $E$ and $V$ can be canonically identified (algebraically) with a subspace of $\operatorname{Lip}_{E^{\prime}}(S, d)^{\prime}$ by $x \otimes \phi(f)=\phi(x \circ f)$, where $x \circ f$ is the element of $\operatorname{Lip}(S, d)$ defined by $x \circ f(s)=\langle x, f(s)\rangle$ for $s \in S$.

From 4.1 and a theorem due to Schatten [10, Theorem 3.2, p. 47] we obtain the basic

Theorem 4.2. Let $(S, d)$ be any metric space and $E$ any Banach space. Then the norm induced on $E \otimes V$ as a subspace of $\operatorname{Lip}_{E^{\prime}}(S, d)^{\prime}$ is the greatest crossnorm $\gamma$.

Let $\lambda$ denote the so-called least crossnorm and $\lambda^{\prime}$ the dual norm.

THEOREM 4.3. The norms $\lambda^{\prime}$ and $\gamma$ agree on $E^{\prime} \otimes \operatorname{lip}^{0}\left(S, d^{\alpha}\right)^{\prime}$ if and only if $\Lambda$ is a surjective isometry and $E \otimes \operatorname{lip}^{0}\left(S, d^{\alpha}\right)$ is dense in lip ${ }_{E}^{0}$ $\left(S, d^{\alpha}\right)$ (under the usual identification $\left.x \otimes f \rightarrow f \cdot x\right)$.

Now, a theorem due to Grothendieck (see [5, Corollary 5.2, p. 110]) gives

Theorem 4.4. Let $E$ be a Banach space, and $0<\alpha<1$. If either $E^{\prime}$ or $\operatorname{lip}^{0}\left(S, d^{\alpha}\right)$ has the approximation property (see [9, Chapter 10, §9]), then $\Lambda$ is a surjective isometry.

When $(S, d)$ is the unit interval, $\operatorname{lip}\left(S, d^{\alpha}\right)^{\prime}$ is isomorphic with $l_{1}$ (see [2]) and therefore has the approximation property. It is not known whether this is true in general.

\section{REFERENCES}

1. R. Arens and J. Eells, Jr., On embedding uniform and topological spaces, Pacific J. Math. 6 (1956), 397-403.

2. Z. Ciesielski, On the isomorphism of the spaces $H_{\alpha}$ and $m$, Bull. Acad. Polon. Sci. Sér. Sci. Math. Astronom. 8 (1960), 217-222. 55-66.

3. K. de Leeuw, Banach spaces of Lipschitz functions, Studia Math. 21 (1961),

4. J. Dixmier, Sur un theorème de Banach, Duke Math. J. 15 (1948), 1057-1071.

5. J. Gil de Lamadrid, Measures and tensors, Trans. Amer. Math. Soc. 114 (1965), 98-121.

6. T. M. Jenkins, Banach spaces of Lipschitz functions on an abstract metric space, Thesis, Yale University, New Haven, Ct. 1967. 
7. R. Phelps, Lectures on Choquet's theorem, Van Nostrand, Princeton, N. J., 1966.

8. A. Roy, Extreme points and linear isometries of the Banach space of Lipschitz functions, Canad. J. Math. 20 (1968), 1150-1164.

9. H. Schaefer, Topological vector spaces, Macmillan, New York, 1966.

10. R. Schatten, $A$ theory of cross-spaces, Ann. of Math. Studies, No. 26, Princeton Univ. Press, Princeton, N. J., 1950.

UNIVERSITY OF ILLINOIS, URBANA, ILLINOIS 61803 